\title{
APLICAÇÃO DA FERRAMENTA DE PROGRAMAÇÃO GNU OCTAVE EM DISCIPLINA DE ESTÁTICA PARA
}

ENGENHARIA

\author{
APPLICATION OF THE GNU OCTAVE PROGRAMMING TOOL IN STATIC DISCIPLINE FOR
}

ENGINEERING

Vitor Hugo Lopes Costa Lima ${ }^{1}$, Rômulo do Nascimento Rodrigues², Roberto de Araújo Bezerra ${ }^{3}$, Pierre Maurice Christophe Lamary ${ }^{4}$

DOI: 10.37702/REE2236-0158.v40p65-75.2021

\section{RESUMO}

Hoje em dia diversas ferramentas já são utilizadas dentro da sala de aula de forma a facilitar o aprendizado. As ferramentas de programação são apresentadas desde o início dos cursos de engenharia, logo são mecanismos que podem muito bem ser utilizados em outras disciplinas, que não necessariamente estejam ligadas à computação. Muitos professores já utilizaram diferentes metodologias com uso de algoritmos em suas salas de aula, nas quais foram proporcionados resultados positivos com relação ao nível de aprendizagem dos alunos. Em muitas dessas aplicações é utilizada a linguagem de programação MATLAB; porém, ela é uma ferramenta paga, o que dificulta seu uso no ambiente acadêmico. Sendo assim, esse trabalho visa a desenvolver uma série de programas didáticos que ajudem no processo de aprendizagem de alunos da disciplina de Estática dos Sistemas Mecânicos de um curso de Engenharia Mecânica a partir da utilização da ferramenta gratuita GNU Octave. Os programas implementados são a solução computacional de alguns problemas apresentados pelo livro didático utilizado na disciplina, empregando-se ferramentas gráficas para ajudar na compreensão. Os programas desenvolvidos ainda serão aplicados à disciplina e serão disponibilizados juntamente com outros manuais da linguagem de programação Octave.

Palavras-chave: MATLAB; Programação; Ensino de Engenharia.

\begin{abstract}
Nowadays various tools are already being used in the class to facilitate the learning. The programming tools are presented since the beginning of engineering courses, so they are mechanisms that may well be used in other subjects that not necessarily are related to computation. A lot of professors have already used different methodology with these algorithms in their classes, where it has provided positive results related to the students learning. In many of these applications it is used the programming language MATLAB, however it is a paid resource, what makes it difficult to use in the academic environment. Therefore, this work aims to develop series of didactic code programs that help in the learning process of the students in a Static of the Mechanical Systems subject, in an Engineering Mechanical course, using the free software GNU Octave. The implemented programs are the computational solution of some problems presented in the textbook used in the subject using graphic tools to help in the comprehension. The developed programs are still going to be applied in the subject and will be available together with other manuals of the programming language Octave.
\end{abstract}

Keywords: MATLAB; Programming; Engineering Teaching.

\footnotetext{
${ }^{1}$ Estudante do Curso de Engenharia Mecânica, Universidade Federal do Ceará, vitorhugo_mec@alu.ufc.br

${ }^{2}$ Prof. Dr. e Vice-coodenador do Curso de Engenharia Mecânica da Universidade Federal do Ceará (UFC), rrodrigues@ufc.br

${ }^{3}$ Prof. Dr. da Universidade Federal do Ceará (UFC), roberto.bezerra@ufc.br

${ }^{4}$ Prof. Dr. da Universidade Federal do Ceará (UFC), pierre.lamary@ufc.br.
} 


\section{INTRODUÇÃO}

Ferramentas computacionais são amplamente utilizadas na engenharia para resolução rápida e segura de diversos problemas, dos mais simples aos mais complexos. Por isso a estrutura curricular de cursos de engenharia já apresenta disciplinas de programação computacional para engenharia desde o primeiro ano, conforme também relatam Manrique e Pávoa (2020). Além disso, atualmente já não é algo incomum um professor universitário lecionar utilizando alguma ferramenta computacional como apoio pedagógico. Como exemplo, Mariani e Martim (2003) aplicaram a resolução de problemas computacionais em disciplinas de Cálculo Numérico, Métodos Numéricos e Álgebra Linear em cursos de engenharia utilizando o software MATLAB. Nas aulas eram mostrados comandos básicos e avançados que poderiam ser utilizados na resolução dos problemas. Castro e Lühmann (2010), Puga (2017) e Manrique e Pávoa (2020) também abordaram o uso de ferramentas de programação no ensino de conteúdos básicos em cursos de engenharia. No último trabalho citado, foi observada pelos autores uma grande aceitação por parte dos alunos envolvidos.

Li e Huang (2017) apresentaram um uso da ferramenta MATLAB aplicada ao ensino em um modelo de sala de aula invertida ${ }^{5} \mathrm{e}$ avaliaram sua efetividade. Os autores também apontaram que o uso de MATLAB para a modelagem de problemas aprimora o processo de aprendizagem. Os autores utilizaram uma metodologia de ensino com videoaulas e depois tira-dúvidas e resolução de problemas em sala de aula. Os autores apontaram a facilidade principalmente para iniciantes no uso do MATLAB, visto que era possível ver as videoaulas quantas vezes os alunos quisessem. O MATLAB já possui uma grande importância no ambiente acadêmico. De fato, a ferramenta já está difundida em diversas instituições de ensino/pesquisa. Um exemplo é a Faculdade de Engenharia da Universidade do Estado do Rio de Janeiro (FEN/UERJ), na qual boa parte dos professores já usam a ferramenta no ensino (DA SILVA; DE LIMA; FERREIRA, 2004).

Castro e Lühmann (2010) apresentam uma aplicação da ferramenta de programação GNU Octave no apoio de aulas. Lessa et al. (2015) desenvolveram uma ferramenta para ajudar na compreensão da lógica de programação de MATLAB e Octave - chamada Octminer, com o auxílio de MVIEs (Múltiplos Ambientes de Visualização Interativa) - que ajuda usuários a entenderem o funcionamento dos códigos fonte.

Deloroso et al. (2019) criaram um material didático para auxiliar alunos de Engenharia Civil por meio de algoritmos escritos na linguagem Octave. A apostila construída aborda problemas referentes a esforços em vigas, Círculo de Mohr, deflexão de vigas e diagrama de momento - carga normal. O trabalho foi desenvolvido por meio do projeto "Pensamento computacional e implementação prática de algoritmos na área de estruturas da construção civil", no Instituto Federal de Santa Catarina, que visava a desenvolver habilidades de programação em alunos de forma a resolver problemas da área. Deloroso et al. (2019) também optaram por usar a linguagem de programação Octave por conta de seu livre acesso, facilidade de uso e compatibilidade com a linguagem MATLAB.

$\mathrm{O}$ uso de softwares com linguagens de programação, apesar de ajudarem no aprendizado e na resolução de problemas, ainda demanda uma carga extra de estudo - o aluno deve aprender e se familiarizar com a linguagem antes de passar para os assuntos mais específicos a serem estudados. Porém, existem ferramentas que ajudam a criar interfaces de interação com o usuário. O trabalho de Santos, Silva e Corrêa (2017) é um exemplo, no qual foi utilizado a plataforma GUI (Graphical User Interface) do MATLAB para se criar uma ferramenta que calcula e mostra os resultados de problemas envolvendo máquinas de indução. A ferramenta criada foi chamada ANAGRIM (Graphical Analysis of Induction Machine). Palhares, Dos Santos e De Vasconcelos (2017) também utilizaram a

\footnotetext{
${ }^{5}$ Bishop e Verleger (2013) apresentam uma abordagem sobre aula invertida, na qual ela é caracteriza por um modelo pedagógico com o uso de solução de problemas e atividades em grupo.
} 
plataforma GUI para problemas envolvendo conceitos específicos de produção. Dessa forma, mesmo usuários que ainda não tinham familiaridade com a linguagem de programação podiam realizar cálculos aplicados ao assunto abordado em aula e ter uma visão mais geral da utilidade da ferramenta.

Vistos todos esses trabalhos utilizando ferramentas de programação como forma de ensino, este presente trabalho almeja apresentar uma metodologia de ensino da disciplina de Estática dos Sistemas Mecânicos para alunos do curso de Engenharia Mecânica na Universidade Federal do Ceará (UFC). Para isso será utilizado a ferramenta GNU Octave, pois esta, além de ser gratuita, possui uma grande compatibilidade com o MATLAB, que já é amplamente utilizado em ambientes acadêmicos. O trabalho foi realizado durante uma Bolsa de Iniciação à Docência fornecida pela Pró-Reitoria de Graduação da UFC.

\section{FERRAMENTAS COMPUTACIONAIS DISPONÍVEIS}

Neste tópico objetiva-se abordar as ferramentas de programação disponíveis mais relevantes para este trabalho. Discute-se um pouco sobre o MATLAB, que já é amplamente utilizada no ambiente de ensino, conforme as citações anteriores comprovam. Também se aborda o GNU Octave. Além destas, restam ainda diversas outras ferramentas de programação disponíveis ${ }^{6}$, inclusive gratuitas, porém optou-se por utilizar GNU Octave.

\section{MATLAB}

MATLAB é um software de programação utilizado para resolver cálculos científicos em engenharia. $O$ termo MATLAB é uma abreviação do termo em inglês Matrix Laboratory; ele foi criado com o intuito de resolver problemas matemáticos simples envolvendo matrizes, porém, com o decorrer do tempo, várias outras funções foram adicionadas e hoje o MATLAB possui uma grande gama de ferramentas para resolver os mais diversos problemas (CHAPMAN, 2003).

Além de possuir uma linguagem simples e fácil, e por ser tão usado ao redor do mundo, o MATLAB possui também muitas referências, tutoriais, manuais e fóruns destinados a ajudar iniciantes e até mesmo programadores avançados da ferramenta ${ }^{7}$. Até mesmo livros didáticos que não estão diretamente ligados à computação já usam essa ferramenta na resolução de problemas matemáticos.

\section{GNU Octave}

GNU Octave é uma ferramenta de programação livre criada para a resolução de problemas numéricos. A sintaxe do Octave é muito similar à sintaxe do MATLAB e, por isso, mesmo não sendo uma ferramenta tão utilizada no meio acadêmico, pode facilmente substituir o MATLAB (LACHNIET, 2020).

Chaves et al. (2016) e Leros, Andreatos e Zagorianos (2010) fizeram algumas comparações utilizando MATLAB e Octave. Ambos os trabalhos chegaram à conclusão de que o Octave é uma boa alternativa ao uso do MATLAB e ainda possui uma performance aceitável, além de todas as semelhanças na sintaxe da linguagem entre Octave e MATLAB. Apesar disso, Leros, Andreatos e Zagorianos (2010) ainda apontaram que o MATLAB é superior em aspectos como velocidade e capacidade gráfica em relação ao Octave.

GNU Octave dispõe de uma ampla biblioteca de pacotes para diversos tipos de estudos que podem ser instalados do site oficial ${ }^{8}$. Graças à sua similaridade com o MATLAB, muito da documentação disponível na internet para o MATLAB também pode ser usada no Octave. Porém, assim como Lachniet (2020) e Lessa et al. (2015) listaram, ainda há algumas funções e sintaxes que não são compatíveis entre as linguagens MATLAB e Octave.

\footnotetext{
${ }^{6}$ Manrique e Pávoa (2020) citam Fortran e C++. Entre outras linguagens pode-se citar Java, Python, C e R.
} 


\section{ESTÁTICA COM OCTAVE}

Dosse et al. (2019) e Ande (2018) são alguns exemplos de trabalhos recentes que visam à aplicação de metodologias de ensino em disciplinas de Estática. Bortolo e Linhares (2006) citaram a disciplina de Estática como umas entre as disciplinas do curso de Engenharia Mecânica que apresentam alto grau de dificuldade de aprendizado e defenderam o uso de dispositivos para facilitar a visualização. Já Steif e Dollár (2005) propuseram a aplicação de dispositivos e ferramentas gráficas para melhorar o ensino dos conceitos de Estáticas.

Bem como a aplicação na engenharia, Da Silva, De Lima e Ferreira (2004) citaram alguns exemplos da aplicação da ferramenta de programação MATLAB em alguns cursos de engenharia. Os autores também mencionaram o uso da ferramenta para resolução de problemas estruturais, o que acarreta uma simplificação, agilidade e precisão nos resultados.

Marghitu, Mihai e Madsen (2013) é um livro didático que aborda Estática utilizando a linguagem MATLAB diretamente, por meio de exemplos e exercícios.

Com o intuito de fornecer uma nova metodologia de ensino para turmas da disciplina de Estática dos Sistemas Mecânicos foi procurada uma maneira de se passar tanto a teoria quanto as resoluções de problemas de Estática.

Os programas preparados como exemplo foram feitos a partir da resolução de problemas de Hibbeler (2005), dos capítulos dois ao nove. De início foram preparados programas a partir da resolução de problemas mais simples que serviriam de base para os alunos. Depois foram preparados outros programas para cada capítulo, alguns envolvendo particularidades dos assuntos abordados, mas ainda bem similares quanto à metodologia das funções e resolução dos problemas.

Os exemplos implementados seguem a mesma metodologia: ao executar o programa o usuário verá um gráfico inicial mostrando o diagrama de corpo livre do problema e poderá dar seus próprios parâmetros pela Janela de Comandos do Octave que plotará outro gráfico bem ao lado, mostrando a diferença entre os dados do usuário e os dados iniciais, por meio dos diagramas de corpo livre. Adicionalmente, é plotada uma tabela comparando os dados e resultados de entrada e os dados e resultados do usuário.

Exemplos foram preparados para que os usuários pudessem modificar os dados de entrada, comparar com os dados originais e verificar a diferença por meio de gráficos ilustrando a direção e intensidade das forças. Os códigos fonte também são todos comentados para que os usuários possam compreender e, posteriormente, reproduzir o que foi feito em outros problemas de Hibbeler (2005).

\section{DESENVOLVIMENTO DOS PROGRAMAS}

Seguindo-se os capítulos de Hibbler (2005) - livro didático utilizado na disciplina -, do segundo ao nono, será apresentada a seguir a sequência dos assuntos abordados nos programas implementados. A estrutura de capítulos abordados é a seguinte: forças vetoriais; equilíbrio de um ponto; resultante de forças; equilíbrio de corpo rígido; análise estrutural; forças internas; atrito; e centro de massa.

\section{Forças vetoriais}

Nesse capítulo são abordados o conceito básico de vetores e as suas operações matemáticas. $\mathrm{O}$ uso do Octave facilita bastante esse passo devido às suas diversas funções de álgebra linear; porém, para os exemplos desenvolvidos apenas as mais simples funções são o suficiente.

Um dos primeiros programas a ser apresentado é baseado em um exemplo de Marghitu, Mihai e Madsen (2013). Foi escolhido esse exemplo inicial, devido à sua versatilidade para demostrar os principais conceitos envolvendo vetores. Nesse exemplo existem quatro vetores bidimensionais: $V_{1}, V_{2}$, $\mathrm{V}_{3}$ e o vetor resultante $\mathrm{V}$, no qual os dados de entrada são a inclinação e intensidade de $\mathrm{V}_{1}$, a intensidade, componente y e ângulo com eixo $\mathrm{x}$ de $V_{2}$ e intensidade de $V_{3}$. O problema pede que se calcule V. Então os estudantes podem modificar esses parâmetros de entrada e comparar os resultados, tendo dessa forma uma 
maneira interativa de visualizar o que acontece com os vetores. A Figura 1 mostra o gráfico resultante com os dados iniciais.

Figura 1 - Exemplo de Vetores 2D

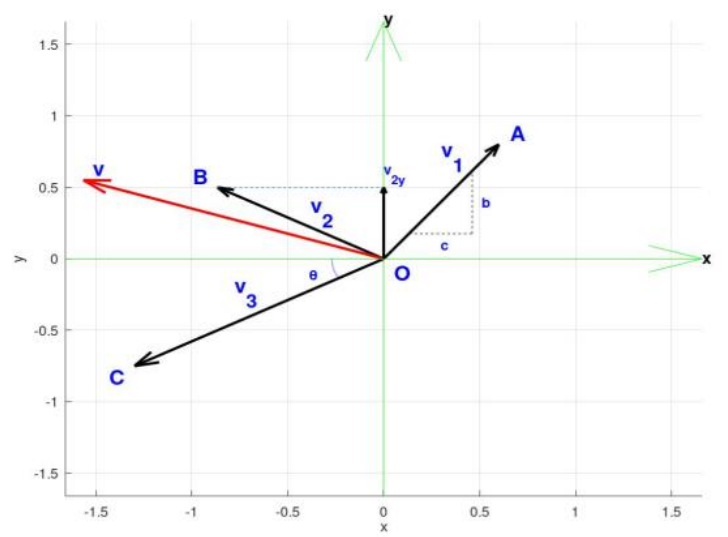

Fonte: elaborada pelos autores.

Outro exemplo que foi implementado teve como base os exemplos de Hibbler (2005) e utilizou vetores tridimensionais. O programa plota um vetor tridimensional $\mathrm{F}$, onde são dados dois ângulos, $\beta$ e $\gamma$, entre o vetor e os eixos $\mathrm{z}$ e $\mathrm{y}$, respectivamente, e se deseja encontrar o valor do ângulo com o eixo $\mathrm{x}, \alpha$, por meio da relação de cossenos dos ângulos. É mostrado na Figura 2 o gráfico resultante com os dados iniciais.

Dessa mesma forma foram implementados outros quatro exemplos com problemas vetoriais 3D com a mesma metodologia de uso. Ao entender o funcionamento desses programas os alunos poderão, além de entender as funções básicas do Octave, entender ferramentas para plotar gráficos (bi e tridimensionais) e funções de álgebra linear simples.

Figura 2 - Exemplo de Vetores 3D

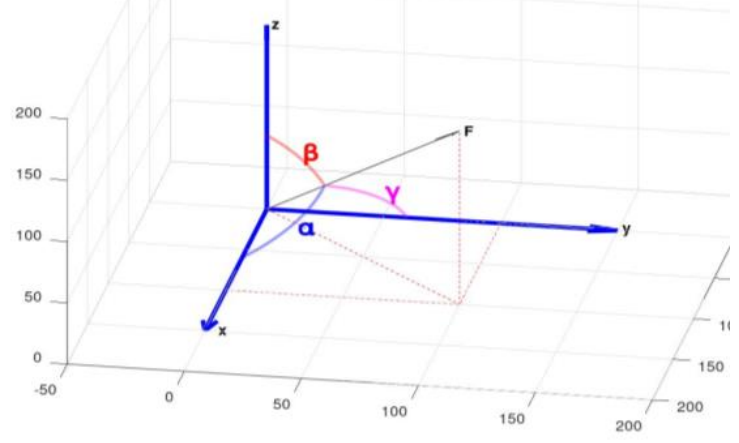

Fonte: elaborada pelos autores.

\section{Equilíbrio de um ponto}

\author{
Figura 3 - Exemplo de equilíbrio de forças em um \\ ponto
}

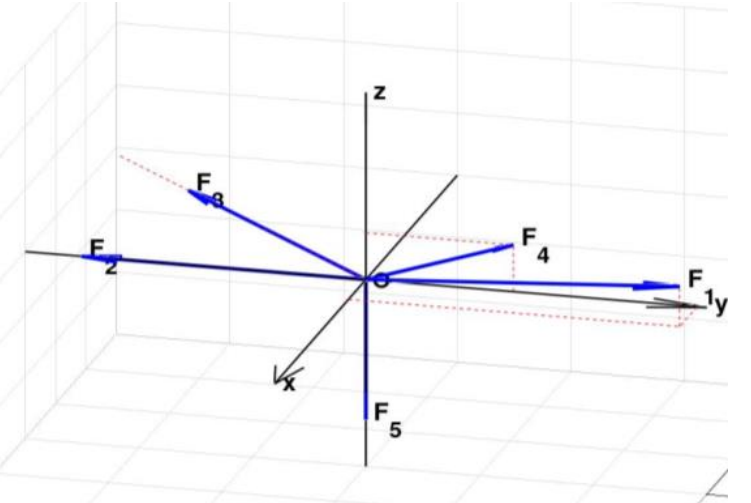

Fonte: elaborada pelos autores.

Esse capítulo do livro de Hibbler (2005) possui em sua teoria geral uma aplicação dos conceitos do capítulo anterior. O conceito base explanado no capítulo é o da regra fundamental de sistemas estáticos: a somatória das forças deve ser igual a zero de maneira a manter o sistema em equilíbrio estático. Foram implementados quatro exemplos a partir de resolução de problemas tridimensionais. Conforme ilustrado na Figura 3, o diagrama de corpo livre de um dos exemplos apresenta um problema em que a força $F_{1}$ deve ser determinada para que o sistema esteja em equilíbrio no ponto de origem $\mathrm{O}$ com as outras 4 forças $-F_{2}, F_{3}, F_{4}$ e $F_{5}$. Os dados de entrada são a intensidade das forças 2 a 5 , a direção de $\mathrm{F}_{3}$ e a inclinação de $\mathrm{F}_{4}$ com o eixo y.

\section{Resultante de forças}

Hibbler (2005) introduz o momento resultante de uma força em seu quarto capítulo. A partir daqui, um sistema estático além de ter uma força resultante igual a zero deve também ter um momento resultante igual a zero. Para sistemas complexos torna-se mais claro como o uso de funções algébricas do Octave agilizam a resolução dos problemas. Foram implementados oito exemplos, sendo um deles com uso de carga distribuída em uma viga. É ilustrado na Figura 4 o resultado de um exemplo em que há uma superfície em forma de 
"Z" inclinada com um momento resultante no eixo $\mathrm{z}$, que deve ser calculado, causado por uma força aplicada no ponto A. Os dados de entrada são as posições $\mathrm{x}$ e y do ponto $\mathrm{A}$, a inclinação da superfície e a intensidade da força aplicada.

Figura 4 - Momento resultante em superfície

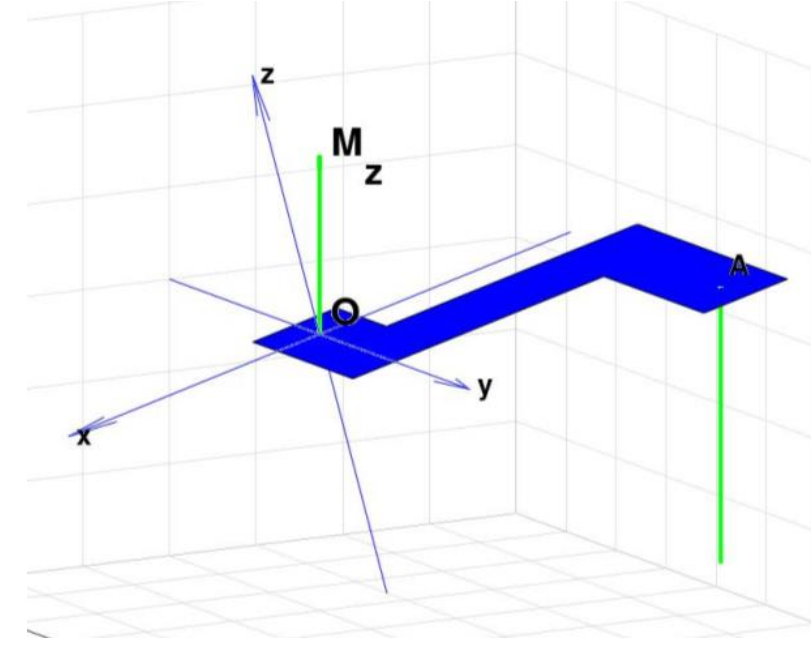

Fonte: elaborada pelos autores.

\section{Equilíbrio de corpo rígido}

No quinto capítulo do livro didático é retratado o equilíbrio de forças em corpos rígidos. O capítulo aborda forças relacionadas a apoios, tanto bi quanto tridimensionais. Geralmente os problemas pedem que se encontre a reação nos apoios, criando-se assim sistemas de equações lineares que podem facilmente ser resolvidos com a utilização de funções algébricas com matrizes do Octave, muito útil quando se trata de um problema mais complexo como em uma viga tridimensional. Foram implementados sete programas. Como é mostrado no diagrama de corpo livre na Figura 5 , um dos problemas implementados pede que se calcule as reações nos apoios, simbolizadas pelas flechas no ponto $\mathrm{C}$ e a reta $\mathrm{AB}$ (em azul), que representa um apoio por cabo. Os dados de entrada são a posição do ponto $\mathrm{A}$, intensidade das duas forças aplicadas $(\mathrm{F} 1$ e F2) e a inclinação do cabo $\mathrm{AB}$. A posição dos nós (ligação entre duas ou mais vigas) da treliça é baseada na posição de entrada de A.

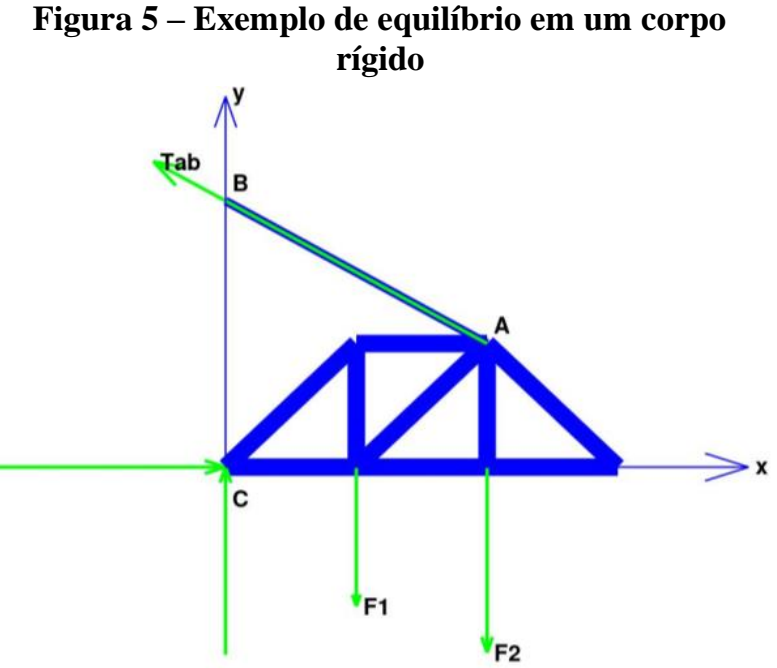

Fonte: elaborada pelos autores.

\section{Análise estrutural}

Para esse tópico do livro são abordadas as forças presente em treliças. A análise visa a identificar as forças de reação em determinado nó de uma treliça e se elas têm efeito de compressão ou tração nas vigas. Nesse capítulo os auxílios de uma linguagem de programação são mais limitados, visto que a maior parte da resolução depende da análise do estudante. Porém, depois de identificada a equação das forças, o processo continua sendo acelerado com a utilização de métodos passados nos capítulos anteriores. Foram implementados cinco programas. $\mathrm{O}$ problema ilustrado aqui apresenta uma treliça apoiada nos pontos $\mathrm{E}$ e $\mathrm{C}$ e com duas forças sendo aplicadas $\left(\mathrm{P}_{1}\right.$ e $\left.\mathrm{P}_{2}\right)$. É pedido que se calcule a força em cada viga e se diga se essa força é de tração ou compressão. Conforme pode se ver no gráfico resultante da Figura 6, as flechas que se encontram dentro das vigas representam uma força compressiva quando estão uma em direção a outra e de tração quando no caso contrário. Os dados que podem ser modificados pelo usuário são a localização $\mathrm{x}$ do ponto $\mathrm{A}$, a localização $\mathrm{x}$ do ponto $\mathrm{B}$ e $\mathrm{C}$ e a intensidade das forças aplicadas. A posição dos outros nós é baseada nas posições dos nós informados. 
Figura 6 - Exemplo de análise estrutural

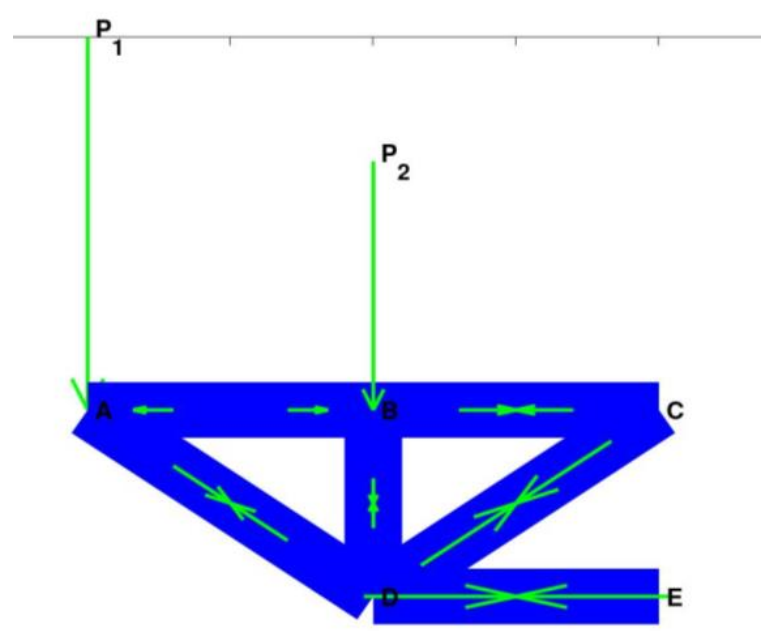

Fonte: elaborada pelos autores.

\section{Forças internas}

Primeiramente, vale ressaltar que esse assunto também foi abordado no trabalho de Deloroso et al. (2019). As forças internas desenvolvidas quando uma força é aplicada em uma viga (ou qualquer corpo rígido) são a Força Cortante (perpendicular à viga), Força Normal (paralela à viga) e o Momento Fletor. As forças internas são conceitos muito importantes em Mecânicas dos Materiais, que ajudam a identificar pontos onde há maior intensidade de esforço em um componente mecânico. Logo, o entendimento dessas ferramentas é de extrema importância para alunos do curso de Engenharia Mecânica e outras engenharias.

Foram implementados cinco exemplos; em três deles, ao contrário dos exemplos anteriores, são plotadas as curvas das funções Força Cortante e Momento Fletor. Conforme pode se observar na Figura 7, um dos exemplos implementados é apresentado graficamente, no qual o problema do livro pede o gráfico de Força Cortante e Momento Fletor em toda a extensão de uma viga sujeita a forças. Ao contrário dos outros exemplos de outros capítulos não há necessidade de uma representação do diagrama de corpo livre, visto que o mais importante é entender a construção dos gráficos. Os dados de entrada são as intensidades das cargas (incluindo forças, momentos e cargas distribuídas) e a localização dos pontos de aplicação.
Figura 7 - Exemplo de forças internas
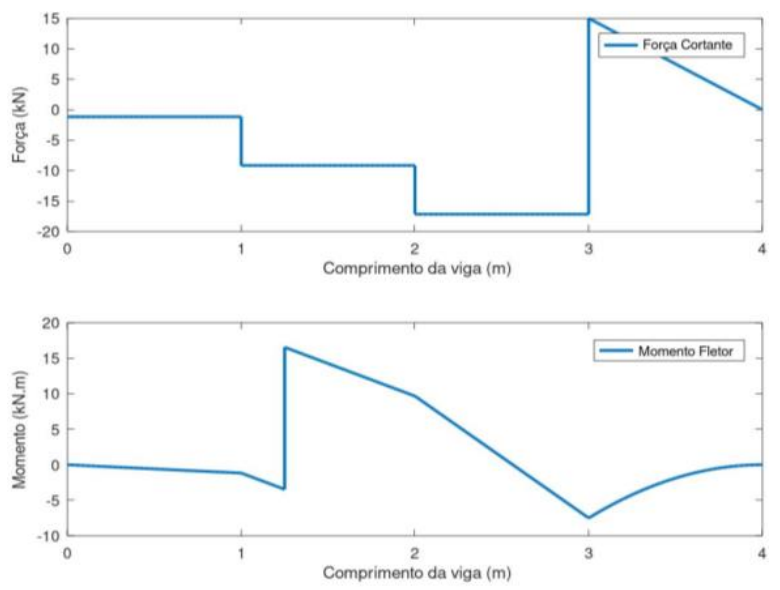

Fonte: elaborada pelos autores.

\section{Atrito}

Esse capítulo do livro didático aborda a definição de forças de resistência ao movimento causadas por atrito. O livro cita o atrito presente em diversos mecanismos, como parafusos, correias, mancais e rolamentos, porém a maioria dos programas implementados foram utilizados somente para resolver problemas mais ilustrativos que permitissem a plotagem de um diagrama de corpo livre de fácil entendimento. Foram implementados cinco exemplos. A partir da Figura 8 pode se observar $\mathrm{o}$ resultado de um exemplo envolvendo duas caixas empilhadas, A e B, e dois rolamentos (pontos $\mathrm{C}$ e D). A caixa B também está presa a um cabo, criando uma tensão $\mathrm{T}_{\mathrm{B}}$. Abaixo da caixa $\mathrm{A}$ pode se ver a representação da força de atrito $\mathrm{F}_{\mathrm{A}} \mathrm{e}$ da força normal $\mathrm{N}_{\mathrm{A}}$. $\mathrm{O}$ problema pede que se calcule a força $\mathrm{P}$ máxima aplicada à corda $\mathrm{DC}$ sem que haja movimento no sistema. Os dados de entrada são o peso das caixas A e B, o comprimento da caixa B e os coeficientes de atrito. Os coeficientes de atrito existentes no sistema são entre as duas caixas, a caixa A com o chão e o atrito nas correias $\mathrm{C}$ e D. A altura da caixa B também é um dado importante para a resolução da questão, mas, para facilitar, a altura de entrada é posta em função do comprimento da caixa quando dada pelo usuário, com a mesma razão dos dados originais. 
Figura 8 - Exemplo de atrito

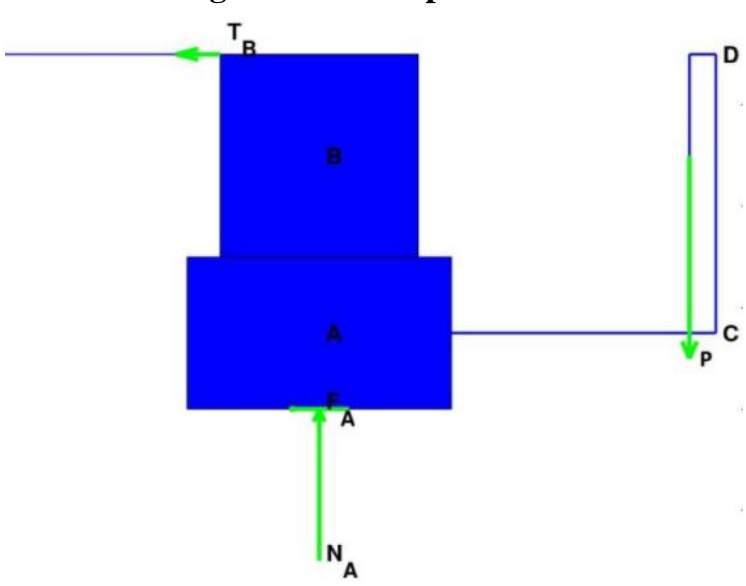

Fonte: elaborada pelos autores.

\section{Centro de Massa}

Até esse ponto o foco de Hibbler (2005) se deu em relação às forças presentes no sistema estático. Porém, nesse capítulo, o foco se dá em calcular a localização do centro de massa de um elemento, sendo esse elemento representado por uma curva, uma superfície ou um sólido. Foram implementados cinco programas. O gráfico resultante de um dos exemplos de centro de massa é ilustrado na Figura 9. Nesse exemplo é pedido que se calcule a posição do centro de massa para um sólido dado na questão por meio de uma função tridimensional. $\mathrm{Na}$ Figura 9 o volume está em um gradiente azulverde $e$ as três superfícies vermelhas retangulares indicam a localização do centro de massa do sólido. O parâmetro de entrada é somente o comprimento máximo da função em y.

Figura 9 - Exemplo de centro de massa

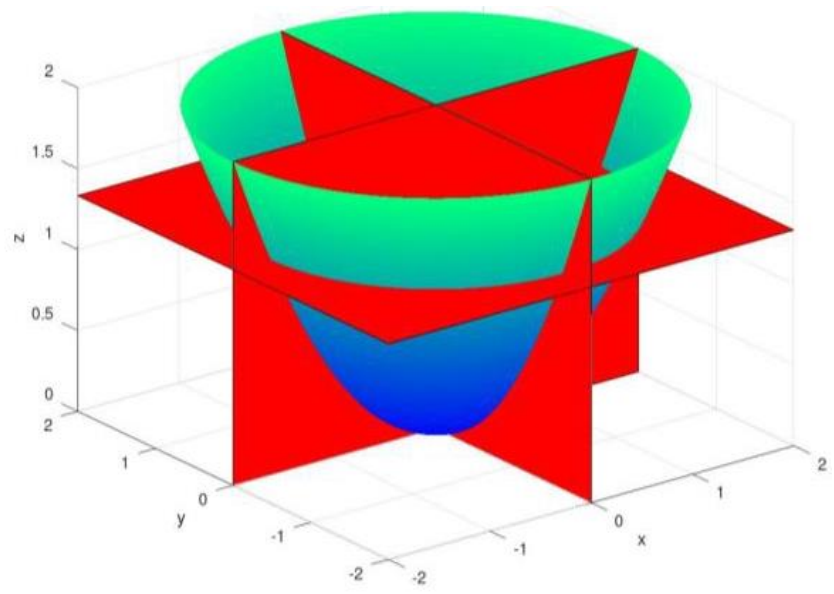

Fonte: elaborada pelos autores.

\section{CONCLUSÕES}

Neste trabalho foram desenvolvidos diversos programas na linguagem Octave abordando problemas de Estática propostos pelo livro didático Hibbeler (2005). Foram ao todo implementados 45 exemplos do livro com o intuito de auxiliar no processo de aprendizagem de alunos de uma disciplina de Estática dos Sistemas Mecânicos. Para tornar os programas didáticos e de fácil entendimento foram utilizadas funções gráficas e vários comentários no código.

Quanto ao uso da linguagem, para alunos iniciantes, é conveniente imaginar que entender os programas implementados pode se tornar um desafio. Por isso também serão disponibilizados manuais do Octave. Uma apostila abordando o uso dos programas será criada e compartilhada com os alunos quando esses programas forem inseridos na disciplina. Além disso, futuramente será implementada uma ferramenta visual pra calcular cargas em vigas e também complementar o entendimento de alunos da disciplina. Outros recursos, como aulas referentes à linguagem de programação, ficarão a cargo do professor caso seja percebida grande dificuldade pelas turmas em implementar os programas.

\section{REFERÊNCIAS}

ANDE, S. Studying the effectiveness of peer instruction in Statics. Proceedings... ASEE GULF-SOUTHWEST SECTION ANNUAL, 2018, Austin. CONFERENCE. Austin: The University of Texas at Austin, 2019.

BISHOP, J. L.; VERLEGER, M. A. The flipped classroom: A survey of the research. Proceedings... ANNUAL CONFERENCE AND EXPOSITION, 120., 2013, Atlanta. [S.l.: s.n], 2013. Disponível em: https://www.researchgate.net/publication/285 935974_The_flipped_classroom_A_survey_of _the_research. Acesso em: 2 ago. 2020.

BORTOLO, K. F.; LINHARES, J. C. Verificação da necessidade de dispositivos didáticos para o ensino na graduação em Engenharia Mecânica. 
Anais... Congresso Brasileiro de Ensino de Engenharia, 34, Passo Fundo, 2006.

CASTRO, E. O.; LÜHMANN, A. C. de O. Software livre aplicado ao ensino de engenharia e computação. Revista de Ciências Exata e Tecnologia. São Paulo, v. 4, n. 4, p. 91-109, dez. 2010.

CHAPMAN, S. J. Programação em MATLAB para Engenheiros. São Paulo: Thomson Learning, 2003.

CHAVES, J. C. et al. Octave and Python: HighLevel Scripting Languages Productivity and Performance Evaluation. Proceedings... HPCMP USERS GROUP CONFERENCE, 6 , [Denver]. [S.l]: IEEE, p. 429-434, 2006.

DA SILVA, J. G. S.; DE LIMA, L. R. O.; FERREIRA, A. R. Uma experiência didática com base no emprego do MATLAB nos cursos de graduação da Faculdade de Engenharia da UERJ. Anais... CONGRESSO BRASILEIRO DE ENSINO DE ENGENHARIA, 32, Brasília, 2004. Disponível em:http://www.abenge.org.br/cobenge/interna .php?ss=15\&ctd=73. Acesso em: 2 ago. 2020.

DELOROSO, J. R. et al. Desenvolvimento de algoritmos como ferramenta de ensinoaprendizagem na área de estruturas. Anais... CONGRESSO BRASILEIRO DE EDUCAÇÃO EM ENGENHARIA, 47. Fortaleza, 2019. Disponível em: http://www.abenge.org.br/sis_artigos.php.

Acesso em: 2 ago. 2020.

DOSSE, L. A. et al. A new approach to teaching Statics using a markerspace. Proceedings... FRONTIERS IN EDUCATION CONFERENCE, 2019, Covington. [S.l.]: IEEE, p. 1-4, 2019. Disponível em: https://ieeexplore.ieee.org/xpl/conhome/90117 01/proceeding. Acesso em: 11 de ago. 2020.

HIBBELER, R. C. Estática Mecânica para Engenharia. 10 ed. São Paulo: Pearson Education, 2005.

LACHNIET, J. Introduction to GNU Octave. 3. ed. Mountain View: Jason Lachniet, 2020. Disponível em: https://www.wcc.vccs.edu/sites/default/files/I
ntroduction-to-GNU-Octave.pdf. Acesso em: 2 ago. 2020.

LEROS, A. P.; ANDREATOS, A., ZAGORIANO, A. Matlab - Octave science and engineering benchmarking and comparison. In: WSEAS INTERNATIONAL CONFERENCE ON COMPUTERS, 14., [2010], [Corfu Island]. Latest Trends on Computers (Volume II). [S.l.]: WSEAS Press, p. 746-754, 2010.

LESSA, I. de M. et al. A Multiple View Interactive Environment to Support MATLAB and GNU/Octave Program Comprehension. Proceedings... INTERNATIONAL CONFERENCE ON INFORMATION TECHNOLOGY: NEW GENERATIONS, 12, 2015, Nevada. [S.l.]: IEEE, p. 552-557, 2015.

LI, X.; HUANG, Z. J. An inverted classroom approach to educate MATLAB in chemical process control. Education for Chemical Engineers, [S.l.], v. 19, p. 1-12, 2017.

MANRIQUE, M. A. A.; PÁVOA, J. M. O papel das ferramentas computacionais avançadas no ensino de engenharia. Revista de Ensino de Engenharia, Brasília, v. 39, p. 3-13, 2020.

MARIANI, V. C.; MARTIM, E. Aplicações do MATLAB no ensino de disciplinas básicas nos cursos de engenharia. Anais... CONGRESSO BRASILEIRO DE EDUCAÇÃO EM ENGENHARIA, 31, Rio de Janeiro, 2003. Disponível em: http://www.abenge.org.br/cobenge/arquivos/1 6/artigos/CBE103.pdf. Acesso em: 2 ago. 2020.

MARGHITU, D. B.; MIHAI, D.; MADSEN, N. H. Statics with MATLAB. London: Springer, 2013.

PALHARES, R. de A.; DOS SANTOS, D. D. da S.; DE VASCONCELOS, N. V. C. Uso de ferramentas computacionais para o auxílio do ensino: $\mathrm{O}$ estudo de caso da Engenharia de Produção. In: COLÓQUIO INTERNACIONAL DE GESTÃO UNIVERSITÁRIA, 17, 2017, Mar del Plata. Florianópolis: Universidade Federal de Santa Catarina, 2017. 
PUGA, L. Z. Sólidos de Revolução num Curso de Engenharia com Matlab. Research, Society and Development. $[S . l]$, v. 4, n. 2, p. 102115, fev. 2017.

SANTOS, N. I. L.; SILVA, S. de O.; CORRÊA, M. B. de R. Desenvolvimento de uma interface gráfica para análise de máquinas elétricas de indução. Anais... CONGRESSO BRASILEIRO DE EDUCAÇÃO EM ENGENHARIA, 45, 2017, Joinville, 2017.
Disponível

http://www.abenge.org.br/sis_artigos.php.

Acesso em: 2 ago. 2020.

STEIF, P. S.; DOLLÁR, A. Reinventing the teaching of Statics. International Journal of Engineering Education, Chicago, v. 21, n. 4, p. 7223-729, 2005 .
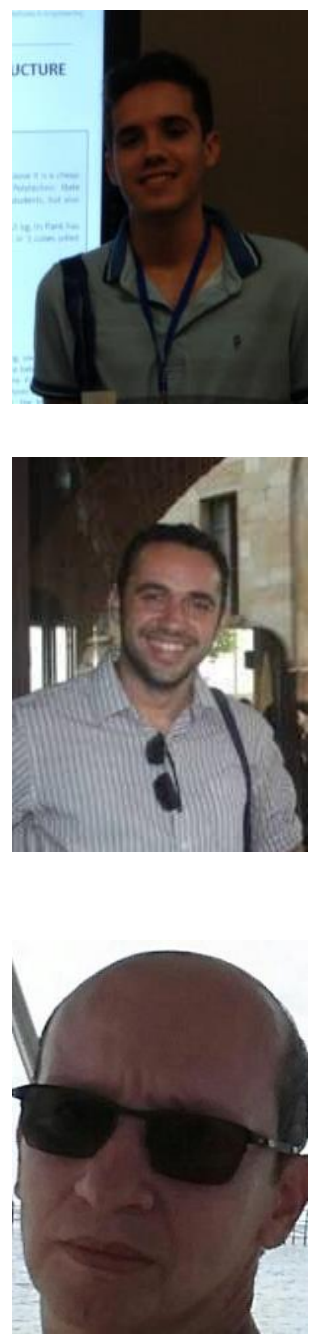

\section{DADOS BIOGRÁFICOS DOS AUTORES}

Vitor Hugo Lopes Costa Lima - Estudante de graduação em Engenharia Mecânica pela Universidade Federal do Ceará (UFC) desde 2016. Bolsista voluntário no Laboratório de Vibrações e bolsista de Iniciação à Docência pela Pró-Reitoria de Graduação da UFC (2019).

Romulo do Nascimento Rodrigues - Graduação em Engenharia Mecânica pela Universidade Federal da Paraíba (2011), Mestrado pelo Programa de PósGraduação em Engenharia Mecânica da Universidade Federal de Campina Grande (2013) e Doutorado em Engenharia Mecânica pela Università di Pisa (Unipi), Pisa, Itália (2017). Atualmente é professor do magistério superior Adjunto da Universidade Federal do Ceará (UFC). Tem experiência na área de Engenharia Mecânica, com ênfase em Elementos de Máquinas, atuando principalmente nos seguintes temas: Tribologia, Vibrações, Análise de Sinais Vibratórios e Elementos Finitos. Atualmente realiza pesquisa nas áreas de Tribologia, Vibrações e análise com elementos finitos.

Roberto de Araújo Bezerra - Graduação em Engenharia Mecânica pela Universidade Federal do Ceará (1990), fez o Mestrado em Engenharia Mecânica pela Faculdade de Engenharia Mecânica de Uberlândia, Minas Gerais (1996) e Doutorado em Engenharia Mecânica pela Universidade Estadual de Campinas Unicamp, São Paulo (2004). Atualmente é professor Associado da Universidade Federal do Ceará. Tem experiência na área de Engenharia Mecânica, com ênfase em Elementos de Máquinas, atuando principalmente nos seguintes temas: Mecanismos, Vibrações, Análise de Sinais Vibratórios e Manutenção Preditiva. 


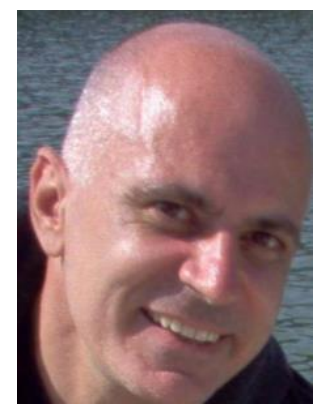

Pierre Maurice Christophe Lamary - Possui graduação em Ciência Aeroespacial e Técnica pela Institut Polytechnique des Sciences Appliquées, science aérospatiale et tec (1986), mestrado em Elementos finitos, Modelização das Estruturas e FD pela Universitè de Technologie de Compiègne (1987), doutorado em Mecânica Aplicada - Acústica pela Universitè de Technologie de Compiègne (1990), pós-doutorado pela Universidade Estadual de Campinas (2005) e pós-doutorado pela Universidade Estadual de Campinas (2010). Atualmente é Professor da Universidade Federal do Ceará. Tem experiência na área de Engenharia Mecânica, com ênfase em Estruturas Aeroespaciais, atuando principalmente nos seguintes temas: Aeronaves, FEM, BEM, Controle de Ruído. 